\title{
Finite time ruin probabilities with one Laplace inversion
}

\author{
Florin Avram ${ }^{\mathrm{a}, \mathrm{b}}$, Miguel Usabel ${ }^{\mathrm{a}, \mathrm{c}, *}$ \\ a Department of Actuarial Mathematics and Statistics, Heriot-Watt University, Edinburgh, UK \\ ${ }^{\mathrm{b}}$ Department of Mathematics, University of Pau, Pau, France \\ ${ }^{\mathrm{c}}$ Dep. de Economia de la Empresa, Universidad Carlos III de Madrid, Campus de Colmenarejo, Comenarejo, Madrid 28270, Spain
}

\begin{abstract}
In this work we present an explicit formula for the Laplace transform in time of the finite time ruin probabilities of a classical Levy model with phase-type claims. Our result generalizes the ultimate ruin probability formula of Asmussen and Rolski [IME 10 (1991) 259] — see also the analog queuing formula for the stationary waiting time of the M/Ph/1 queue in Neuts [Matrix-geometric Solutions in Stochastic Models: An Algorithmic Approach. Johns Hopkins University Press, Baltimore, MD, 1981] — and it considers the deficit at ruin as well.
\end{abstract}

JEL classification C690; C220

Keywords Finite-time ruin probability; Phase-type distribution; Deficit at ruin; Lundberg's equation; Laplace transform

\section{Introduction}

\subsection{The model}

We consider a classical risk process in continuous time $\left\{U_{t}\right\}_{t \geq 0}$ with $Z_{k}$ claim sizes and premium $c$ per time unit,

$$
U_{t}=u+c t-\sum_{k=1}^{N_{t}} Z_{k},
$$

where $u$ are the initial reserves and $N_{t}$ the total number of claims up to time $t$. The claim times sequence forms a Poisson process with interarrival times exponentially distributed with density $w(t)=\lambda \mathrm{e}^{-\lambda t}$. We will assume that the claim sizes $Z_{k}$ have a phase-type distribution (see next section) $F(z)$, with density and Laplace transform denoted by $f(z), f^{*}(s)$. The Levy exponent of the process $\kappa(s)$ defined by $\mathbb{E} \mathrm{e}^{s U_{t}}=\mathrm{e}^{t \kappa(s)}$ is given by

$$
\kappa(s)=c s+\lambda\left(f^{*}(s)-1\right)
$$

* Corresponding author. Present address: Dep. de Economia de la Empresa, Universidad Carlos III de Madrid, Campus de Colmenarejo, Comenarejo, Madrid 28270, Spain.

E-mail addresses florin.avram@univ-Pau.fr (F. Avram), usabel@emp.uc3m.es (M. Usabel). 


\subsection{The ruin problem}

Let $\tau=\inf \left\{t>0: U_{t}<0\right\}$ be the ruin time and $Y=-U_{\tau}$ be the deficit at ruin or severity of ruin. We are interested in the probability of ruin with time span $t$ and initial reserves $u$ and severity of ruin larger than $y$, i.e.

$$
\Psi_{t, u, y}=\mathbb{P}_{u}\{\tau\langle t, Y\rangle y\}
$$

We will study its density with respect to $t, \psi_{t, u, y}=\partial \Psi_{t, u, y} / \partial t$, and its more tractable Laplace transform in time:

$$
\psi_{a, u, y}^{*}=\int_{0}^{\infty} \mathrm{e}^{-a t} \psi_{t, u, y} \mathrm{~d} t .
$$

This function satisfies a second kind Volterra integro-differential equation. One natural approach to this kind of problems, going back in this case to Cramèr (1957) and Sparre Andersen (1957), consists in taking a double Laplace-Stieltjes transform of the equation of interest (or triple, if the deficit $Y$ is also included), to be inverted numerically. The results contained in Thorin (1971) show formulae for the mentioned double Laplace transforms. For alternative classical approaches, see for example the pioneer works by Arfwedson (1954) who uses the saddle-point approximation and Seal (1974) who uses differential equations.

\section{3. "Phase-type" modeling}

It is well known that simplifications in the resolution of second kind Volterra integro-differential equations are possible if the kernel is degenerate, i.e. a finite sum of products (for example, the equation becomes equivalent to a linear ordinary differential system). Asmussen and Rolski (1991) and Asmussen (1992) proposed (following analog work in queuing theory), a probabilistic alternative to this analytic approach, based upon assuming that the claims and interrivals time have phase-type distributions. These are defined as probability distributions of matrix-exponential type (see below), and they have also an important probabilistic interpretation as absorption times of finite (transient) homogeneous Markov processes to a special absorbing state called cemetery.

More precisely, let $J_{t}$ denote a terminating Markov process with finite state space $E=\{C, 1, \ldots, m\}$, where $C$ is an absorbing state (i.e. $\mathbb{P}_{i}\left[J_{t}=C\right.$ eventually $]=1$ for any state $i$ ) and let $\xi=\inf \left\{t>0: J_{t}=C\right\}$ denote the absorption time to the cemetery. Let $\mathbf{G}$ denote the restriction of the intensity matrix of $J_{t}$ to $\{1, \ldots, m\}$, and note that the rates of absorption to the cemetery are given by the column vector $\mathbf{g}=-\mathbf{G} \mathbf{1}$, where $\mathbf{1}=(1 \ldots 1)^{\prime}(\mathrm{a}$ column vector). Let $\alpha$ denote the initial distribution of $J_{0}$, written as a row vector. Then, it is easy to check that the distribution of $\xi$ satisfies:

$$
\begin{aligned}
& F(x)=\mathbb{P}_{\boldsymbol{\alpha}}(\xi \leq x)=1-\boldsymbol{\alpha} \mathrm{e}^{\mathbf{G} x} \mathbf{1}, \\
& f(x)=\boldsymbol{\alpha} \mathrm{e}^{\mathbf{G} x} \mathbf{g},
\end{aligned}
$$

and

$$
f^{*}(s)=\mathbb{E}_{\boldsymbol{\alpha}} \mathrm{e}^{-s \xi}=\int_{0}^{\infty} \mathrm{e}^{-s x} F(\mathrm{~d} x)=\boldsymbol{\alpha}(s \mathbf{I}-\mathbf{G})^{-1} \mathbf{g} .
$$

Definition. A probability distribution $F(x)$ is said to be of phase-type $(\alpha, G, m)$ if it is of the form (5), for some probability vector $\alpha$ and some matrix $G$ which is a subintensity matrix (i.e. with nonnegative off diagonal elements and nonpositive row sums).

Phase-type distributions include and generalize exponential distributions in series and/or parallel. They have found numerous applications in applied probability and are of considerable practical interest, since they form a dense class in the set of all distributions on $(0, \infty)$. 
The advantages of this class of distributions can be perceived from several points of view:

- Algebraic and numerical convenience due to the possibility of manipulations of matrix exponentials, which generalize the scalar exponential case $(n=1)$. Some of the first are illustrated in this paper; the latter may be seen in the companion paper of Asmussen et al. (2002), where a numerical method for computing finite time ruin probabilities without Laplace inversion is proposed and implemented.

- The availability of probabilistic interpretations of the results. For the interpretation of our result, which basically says that with phase-type jumps, the Laplace transform in time of the ruin probability has a similar phase-type formula, see Asmussen (2000), and Appendix A. For now, we note that it is a consequence of the property that the conditional overshoot distribution $F(x+y) /(1-F(x))$ belongs to a finite vector space. More precisely, this overshoot distribution is again phase-type with the same $m$ and $\mathbf{G}$, but with $\alpha_{i}$ replaced by $\operatorname{Pr}\left(J_{x}=i \mid \zeta>x\right)$, which is reminiscent of the memoryless property of the scalar exponential distribution.

We note that while the convenience of phase-type distributions had long been recognized for the perpetual ruin problem, it is only recently that it has started being implemented for computing finite time ruin probabilities. For example, related in spirit to our work are the papers of Stanford and Stroinski (1994, 2000) (who use a recursive approach) and Willmot (1999, 2000).

\subsection{The result}

The motivation of our result, which generalizes the perpetual ruin formula of Asmussen and Rolski (1991) see (13) - comes from the observation that the Laplace transform in time $\psi_{a, u, y}^{*}$ coincides also with the expected probability of ruin before an exponential horizon $H_{a}$ with parameter $a$, independent of the process. Indeed, an integration by parts shows that:

$$
\begin{aligned}
& \psi_{a, u, y}^{*}=\int_{0}^{\infty} \mathrm{e}^{-a t} \psi(u, t, y) \mathrm{d} t=\int_{0}^{\infty} a \mathrm{e}^{-a t} \mathbb{P}_{u}\{\tau<t ; Y>y\} \mathrm{d} t, \\
& \psi_{a, u, y}^{*}=\mathbb{P}_{u}\left\{\tau<H_{a} ; Y>y\right\}=\mathbb{E} \Psi\left(H_{a}, u, y\right) .
\end{aligned}
$$

Furthermore, an exponential horizon $H_{a}$ is probabilistically similar to a perpetual horizon, since by the memoryless property of the exponential, the passage of time does add any extra information. Thus, in principle every perpetual result should have an extension to an exponential horizon result; however, as our result illustrates (see (10) and (11)), the extension may not be altogether obvious.

Theorem 1. If $U_{t}$ is a classical risk process (1) with claim sizes of phase-type (p, G) and a $>0$ is a positive number, then:

(a) The single Laplace transform in the time tof the ruin function (4) (with given initial reserves $u$ and deficit at ruin greater than $y$ ) is

$$
\psi_{a, u, y}^{*}=\mathbf{p}_{a} \mathrm{e}^{\mathbf{Q}_{a} u} \mathrm{e}^{\mathbf{G} y} \mathbf{1},
$$

where

$$
\mathbf{p}_{a}=\left(\frac{\lambda}{c}\right) \mathbf{p}\left(s_{a} \mathbf{I}-\mathbf{G}\right)^{-1}, \quad \mathbf{Q}_{a}=\mathbf{G}+\mathbf{g p}_{a},
$$

and $s_{a}$ is the (unique) nonnegative root of the Lundberg equation

$$
\kappa\left(s_{a}\right)=a .
$$


(b) The double Laplace transform of the ruin time $\tau$ and of the ruin deficit $Y$ (i.e. the transform in $t, y$ of the joint density $\left.-\partial \psi_{t, u, y} / \partial y\right)$ is given by

$$
\psi_{a, u, b}^{* *}=-\int_{0}^{\infty} \int_{0}^{\infty} \frac{\partial \psi_{t, u, y}}{\partial y} \mathrm{e}^{-a t-b y} \mathrm{~d} t \mathrm{~d} y=\mathbb{E}_{u} \mathrm{e}^{-a \tau-b Y}=\mathbf{p}_{a} \mathrm{e}^{\mathbf{Q}_{a} u}(b \mathbf{I}-\mathbf{G})^{-1} \mathbf{g} .
$$

Notes.

(1) Parts (a) and (b) may be seen to be equivalent by taking the Laplace transform in $y$ of the derivative in $y$ of (10), i.e. $\mathbf{p}_{a} \mathrm{e}^{\mathbf{Q}_{a} u} \mathrm{e}^{\mathbf{G} y} \mathbf{g}$.

(2) When $a=0$ (thus $s_{a}=0$ ) and $y=0$, part (a) reduces to the perpetual ruin formula 3.1 of Asmussen and Rolski (1991)

$$
\psi_{0, u, 0}^{*}=\left(\frac{\lambda}{c}\right) \mathbf{p}(-\mathbf{G})^{-1} \mathrm{e}^{\mathbf{Q} u} \mathbf{1}
$$

The case $y=0$ and $a>0$ yields the formula

$$
\psi_{a, u, 0}^{*}=\mathbf{p}_{a} \mathrm{e}^{\mathbf{Q}_{a} u} \mathbf{1}=\left(\frac{\lambda}{c}\right) \mathbf{p}\left(s_{a} \mathbf{I}-\mathbf{G}\right)^{-1} \mathrm{e}^{\mathbf{Q}_{a} u} \mathbf{1},
$$

which states that the killed ruin probability inherits a phase-type representation $\left(\mathbf{p}_{a}, \mathbf{Q}_{a}\right)$ from the claims, with $\mathbf{Q}_{a}=\mathbf{G}+\mathbf{g p}_{a}$. This special form of the intensity matrix $\mathbf{Q}_{a}$ and the fact that the conditional surplus distribution remains unchanged with phase-type jumps have a nice probabilistic interpretation offered in Asmussen (2000, Chapter 8, Corollary 3.1) (included for convenience in Appendix A). The formula (11) for the vector $\mathbf{p}_{a}$ offered here is however new. For a generalization to the case of arbitrary phase-type interarrivals, see Avram and Usabel (2003).

(3) An alternative "spectral expansion" formula for the single Laplace transform was obtained by Thorin (1971, Formula 4.4) (for hyperexponential claim sizes). However, Thorin's formula requires computing all the negative roots of the Lundberg equation, which it also assumes to be distinct. Our formula uses instead the unique nonnegative root (independently of the complexity of the phase-type representation), without any additional assumptions. We view these two points as its major practical contribution.

(4) Gerber and Shiu (1997, 1998)—see also Gerber and Landry (1998) for an interesting paper incorporating Brownian motion in the model as well-have obtained explicit formulas requiring one numerical integration for the function $\Psi_{a, 0, y}^{*}$ which do not assume phase-type jumps. However, to deal with positive surpluses $u>0$, they still need to solve one renewal equation which requires a second Laplace transform in $u$.

\subsection{Example}

In the exponential case with claim intensity $\mu$, which is of phase-type with $\mathbf{p}=1, \mathbf{G}=-\mu$ we find that the single Laplace transform is

$$
\psi_{a, u, y}^{*}=\frac{\lambda}{\mu+s_{a}} \mathrm{e}^{-\mu\left(1-\lambda /\left(\mu+s_{a}\right)\right) u} \mathrm{e}^{-b y},
$$

where $s_{a}$ is the nonnegative root of the quadratic equation $\kappa(s)=s(1-\lambda /(s+\mu))=a$. This is a famous text-book formula; see for instance Gerber et al. (1987). For example, when $a=y=0$ this becomes $\psi_{0, u, 0}^{*}=(\lambda / \mu)$ $\mathrm{e}^{-(\mu-\lambda) u}$. 


\subsection{Contents}

The approach for establishing Theorem 1 consists in applying a further Laplace transform in $u$. Some special properties of phase-type distributions-reviewed in the next section-will allow us to invert the transforms (except for the one in $t$ ). The proof of the theorem is given in Section 3.

\section{Some auxiliary results}

We present now a classical "fluctuation theory" result for the triple Laplace transform of the multivariate ruin density

$$
\psi_{a, s, b}^{* * *}=\int_{0}^{\infty} \mathrm{e}^{-s u} \mathbb{E}_{u} \mathrm{e}^{-a \tau-b Y_{\tau}} \mathrm{d} u=\int_{0}^{\infty} \mathrm{e}^{-s u} \psi_{a, u, b}^{* *} \mathrm{~d} u
$$

for spectrally one-sided processes. An expression for it may be found for example in Bingham (1975, Theorem 6a) or Bertoin (1996); we use instead a simplified expression given in Avram et al. (2002, Corollary 2.3):

Proposition 1. The triple Laplace transform (14) of the ruin function is

$$
\psi_{a, s, b}^{* * *}=(a-\kappa(s))^{-1}\left(\frac{\kappa(s)-k(b)}{b-s}-\frac{\kappa\left(s_{a}\right)-\kappa(b)}{b-s_{a}}\right) .
$$

A simplification in the phase-type case will come from an analog of the identity in Asmussen and Bladt (1997, Lemma 3.1).

\section{Proposition 2.}

$$
\frac{\mathbf{p}_{*}(s \mathbf{I}-\mathbf{G})^{-1} \mathbf{v}_{1}}{1-\mathbf{p}_{*}(s \mathbf{I}-\mathbf{G})^{-1} \mathbf{v}}=\mathbf{p}_{*}\left(s \mathbf{I}-\mathbf{G}-\mathbf{v} \mathbf{p}_{*}\right)^{-1} \mathbf{v}_{1} .
$$

Proof. We will apply to the RHS $\mathbf{p}_{*}\left(s \mathbf{I}-\mathbf{G}-\mathbf{v} \mathbf{p}_{*}\right)^{-1} \mathbf{v}_{1}$ a well-known matrix identity (see for example Seber (1984, p. 519))

$$
\left(\mathbf{A}-\mathbf{v} \mathbf{p}_{*}\right)^{-1}=\mathbf{A}^{-1}+\mathbf{A}^{-1} \mathbf{v}\left(1-\mathbf{p}_{*} \mathbf{A}^{-1} \mathbf{v}\right)^{-1} \mathbf{p}_{*} \mathbf{A}^{-1}
$$

with $\mathbf{A}=(s \mathbf{I}-\mathbf{G})$. This yields:

$$
\begin{aligned}
\mathbf{p}_{*}\left(s \mathbf{I}-\mathbf{G}-\mathbf{v} \mathbf{p}_{*}\right)^{-1} \mathbf{v}_{1} & =\mathbf{p}_{*}\left(\mathbf{A}-\mathbf{v} \mathbf{p}_{*}\right)^{-1} \mathbf{v}_{1} \\
& =\mathbf{p}_{*}\left(\mathbf{A}^{-1}+\mathbf{A}^{-1} \mathbf{v}\left(1-\mathbf{p}_{*} \mathbf{A}^{-1} \mathbf{v}\right)^{-1} \mathbf{p}_{*} \mathbf{A}^{-1}\right) \mathbf{v}_{1}=\sigma_{2}+\frac{\sigma_{1} \sigma_{2}}{1-\sigma_{1}}=\frac{\sigma_{2}}{1-\sigma_{1}},
\end{aligned}
$$

where $\sigma_{2}=\mathbf{p}_{*} \mathbf{A}^{-1} \mathbf{v}_{1}$ and $\sigma_{1}=\mathbf{p}_{*} \mathbf{A}^{-1} \mathbf{v}$.

Note. This identity is useful when a Laplace transform may be expressed in the form of the LHS above; in that case, the RHS provides a matrix-exponential representation for the density, and relieves us therefore of the need to invert the Laplace transform.

\section{Proof of the theorem}

Proof. Part (a) is clearly equivalent to part (b); it is enough therefore to establish the latter. We simplify first the triple Laplace transform (15), using $\kappa(s)=c s+\lambda\left(\mathbf{p}(s \mathbf{I}-\mathbf{G})^{-1} \mathbf{g}-1\right)$ and the resolvent identity:

$$
(s \mathbf{I}-\mathbf{G})^{-1}-\left(s^{\prime} \mathbf{I}-\mathbf{G}\right)^{-1}=\left(s^{\prime}-s\right)(s \mathbf{I}-\mathbf{G})^{-1}\left(s^{\prime} \mathbf{I}-\mathbf{G}\right)^{-1} .
$$


We find that:

$$
\frac{\kappa(s)-\kappa(b)}{b-s}=-c+\lambda \frac{\mathbf{p}\left((s \mathbf{I}-\mathbf{G})^{-1}-(b \mathbf{I}-G)^{-1}\right) \mathbf{g}}{b-s}=-c+\lambda \mathbf{p}(s \mathbf{I}-\mathbf{G})^{-1}(b \mathbf{I}-\mathbf{G})^{-1} \mathbf{g} .
$$

Plugging this in (15), using again the resolvent identity and putting $\mathbf{v}_{1}=(b \mathbf{I}-\mathbf{G})^{-1}$ yields:

$$
\begin{aligned}
\psi_{a, s, b}^{* * *} & =\frac{1}{a-\kappa(s)}\left(\lambda \mathbf{p}(s \mathbf{I}-\mathbf{G})^{-1}(b \mathbf{I}-\mathbf{G})^{-1} \mathbf{g}-\lambda \mathbf{p}\left(s_{a} \mathbf{I}-\mathbf{G}\right)^{-1}(b \mathbf{I}-\mathbf{G})^{-1} \mathbf{g}\right) \\
& =\frac{1}{a-\kappa(s)} \lambda \mathbf{p}\left((s \mathbf{I}-\mathbf{G})^{-1}-\left(s_{a} \mathbf{I}-\mathbf{G}\right)^{-1}\right)(b \mathbf{I}-\mathbf{G})^{-1} \mathbf{g} \\
& =\frac{s_{a}-s}{\left.\kappa\left(s_{a}\right)-\kappa(s)\right)} \lambda \mathbf{p}(s \mathbf{I}-\mathbf{G})^{-1}\left(s_{a} \mathbf{I}-\mathbf{G}\right)^{-1}(b \mathbf{I}-\mathbf{G})^{-1} \mathbf{g} \\
& =\frac{\lambda \mathbf{p}(s \mathbf{I}-\mathbf{G})^{-1}\left(s_{a} \mathbf{I}-\mathbf{G}\right)^{-1}(b \mathbf{I}-\mathbf{G})^{-1} \mathbf{g}}{c-\lambda \mathbf{p}(s \mathbf{I}-\mathbf{G})^{-1}\left(s_{a} \mathbf{I}-\mathbf{G}\right)^{-1} \mathbf{g}} \\
& =\frac{\mathbf{p}_{a}(s \mathbf{I}-\mathbf{G})^{-1} \mathbf{v}_{1}}{1-\mathbf{p}_{a}(s \mathbf{I}-\mathbf{G})^{-1} \mathbf{g}}=\mathbf{p}_{a}\left(s \mathbf{I}-\left(\mathbf{G}+\mathbf{g p}_{a}\right)^{-1} \mathbf{v}_{1},\right.
\end{aligned}
$$

where the last equality comes from Proposition 2.

Finally, we recognize the last form to be the Laplace transform in $u$ of the function $\mathbf{p}_{a} \mathrm{e}^{\left(G+\mathbf{g} \mathbf{p}_{a}\right) u}(b \mathbf{I}-\mathbf{G})^{-1} \mathbf{g}$.

\section{Conclusions}

In conclusion, although Theorem 1 is mathematically quite close to previous known results, it does yield a simpler formula for the Laplace transform in time of the finite time ruin probabilities than Thorin (1971), with less assumptions (see note (3) after the theorem), and including the deficit at ruin as well. In conclusion, our result suggests two possible approaches for calculating/approximating the finite time ruin probabilities:

- Inverting the Laplace transform in time (since there is only one transform to invert, there will be of course less numerical errors).

- Using random horizon approximations. A first rough approximation for a fixed horizon $t$ may be obtained by choosing an exponential horizon $H_{a}$ with expectation $t$ (thus $a=t^{-1}$ ). This replaces the finite time ruin probability by the rough approximation $t^{-1} \psi_{t^{-1}, u, y}^{*}$ which is however obtained via only one evaluation of the explicitly available transform (thus, no inversion)!

Further (converging) approximations may be obtained by choosing Erlang random horizons with expectation equal to the desired fixed one $t$, as proposed and implemented in Asmussen et al. (2002) (also, with no inversion).

The second approach is may be less attractive nowadays than it would have been twenty years ago, before the developing of effective numerical inversion methods (like that of Gaver-Stehfest, for example). Its potential is worth however further investigation.

\section{Acknowledgements}

This paper benefited from discussions with Soren Asmussen, and from useful comments from the referee. This research was partially funded by MEyC Proyecto SEC2001-1169. 


\section{Appendix A}

Formula (10) comes with a convenient probabilistic interpretation—see for example Asmussen (2000, pp. 227-231), obtained by following the distribution of the Markovian phase process $\tilde{J}_{t}$ which gives the phase of the jump at the first visit time of any point $t$, while $t$ moves from $u$ to 0 . Let $\mathbf{p}_{a}$ denote the row probability vector giving the "initial" distribution of the phases at the first descending ladder height time of the risk process, obtained before the exponential random time horizon $H_{a}$.

Note then that there are two ways for the process $\tilde{J}_{t}$ to change phase: one, describing the phase changes "within jump" at rates G, and the second describing phase changes after the end of a jump and passing through "an invisible upward excursion". The end of a jump occurs at rates $\mathbf{g}$, and the start of the next downwards ladder point following

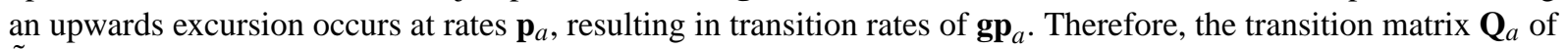
$\tilde{J}_{t}$ must equal $\mathbf{G}+\mathbf{g p}_{a}$.

Finally, note that below 0 and until the ruin deficit $-Y$, the phase process $\tilde{J}_{t}$ continues simply at rate $\mathbf{G}$, implying therefore that the deficit distribution is phase type $\left(\mathbf{p}_{a} \mathrm{e}^{\mathbf{Q}_{a} u}, \mathbf{G}\right)$.

Notes.

(1) The easiness with which the deficit result may be obtained under phase-type assumptions may be viewed as one of the main advantages of this approach.

(2) This probabilistic structure turns out to be especially important in Asmussen et al. (2002), for the solution of the Erlang killed case.

\section{References}

Arfwedson, G., 1954. Research in collective risk theory. Skandinavisk Aktuarietidskrift 37, 191-223.

Asmussen, S., 1992. Phase-type representations in random walk and queueing problems. The Annals of Probability 20, 772-789.

Asmussen, S , 2000. Ruin Probabilities. World Scientific, Singapore.

Asmussen, S., Bladt, M., 1997. Renewal theory and queueing algorithms for matrix-exponential distributions. Chakravarthy, S.R., Alfa, A.S. (Eds.), Matrix-analytic Methods in Stochastic Models. Marcel Dekker, New York, pp. 313-341.

Asmussen, S., Rolski, 1991. Computational Methods in risk theory: a matrix-algorithmic approach. IME 10, 259-274.

Asmussen, S., Avram, F., Usabel, M., 2002. Erlangian approximations for finite-horizon ruin probabilities. Astin Bulletin 32, $267-281$.

Avram, F., Usabel, M, 2003. Ruin probabilities for risk processes with phase-type waiting times. Submitted for publication.

Bertoin, J., 1996. Lévy Processes. Cambridge University Press, Cambridge.

Bingham, N.H., 1975. Fluctuation theory in continuous time. Advances in Applied Probability 7, 705-766.

Cramèr, H., 1957. Collective Risk Theory. Jub. Volume of F. Skandia.

Gerber, H., Landry, B., 1998. On the discounted penalty at ruin in a jump-diffusion and the perpetual put option. Insurance: Mathematics and Economics 22, 263-276.

Gerber, H., Shiu, E., 1997. The joint distribution of the time to ruin, the surplus immediate before ruin, and the deficit at ruin. Insurance: Mathematics and Economics 21, 129-137.

Gerber, H., Shiu, E., 1998. On the time value of ruin. North American Actuarial Journal 2 (1), 48-78.

Gerber, H., Goovaerts, M., Kaas, R., 1987. On the probability and severity of ruin. ASTIN Bulletin 17 (2), 151-163.

Seal, H., 1974. The numerical calculation of $U(w, t)$, the probability of non-ruin in an interval $(0, t)$. Scandinavian Actuarial Journal 1974 , 121-139.

Seber, G.A.F., 1984. Multivariate Observations. Wiley, New York.

Sparre Andersen, E., 1957. On the collective theory of risk in the case of contagion between claims. In: Proceedings of the Transactions of the XVth International Congress on Actuaries, vol. II, New York, pp. 219-229.

Stanford, D., Stroinski, K., 1994. Recursive methods for computing finite-time ruin probabilities for phase-type distributed claim sizes. Astin Bulletin 24 (2), 235-254.

Stanford, D., Stroinski, K., Lee, K., 2000. Ruin probabilities based at claim instants for some non-Poisson claim processes. Insurance: Mathematics and Economics 26, 251-267.

Thorin, O., 1971. Further remarks on the ruin problem in case the epochs of claims form a renewal process. Skandinavisk Aktuarietidskrift 1438, 121-142.

Willmot, G., 1999. A Laplace transform representation in a class of renewal queueing and risk process. Journal of Applied Probability 36, $570-584$.

Willmot, G., 2000. On the evaluation of the conditional distribution of the deficit at the ruin time. Scandinavian Actuarial Journal 100 (1), 63-79. 